\title{
The effect of noise reduction in measuring the linear and nonlinear dependency of financial markets
}

\author{
Hossein Hassani ${ }^{\mathrm{a}, \mathrm{b}, *}$, Andreia Dionisio $^{\mathrm{c}}$, Mansoureh Ghodsi ${ }^{\mathrm{b}}$ \\ ${ }^{a}$ Institute for Studies and Research (ITST), Tehran, Iran \\ ${ }^{\mathrm{b}}$ Centre for Optimisation and Its Applications, School of Mathematics, Cardiff University, CF24 4AG, UK \\ ${ }^{\mathrm{c}}$ University of Evora, CEFAGE-UE, Largo Colegiais, 2, 7000 Evora, Portugal
}

\section{A R T I C L E I N F O}

\section{Article history:}

Received 4 April 2008

Accepted 6 January 2009

\section{Keywords:}

Measure of dependencies

Noise reduction

Stock markets

Mutual information

Detrended fluctuation analysis

Detrended moving average method

Singular spectrum analysis

ARMA

GARCH

\begin{abstract}
A B S T R A C T
The daily closing prices of several stock market indices are examined to analyse whether noise reduction matters in measuring dependencies of the financial series. We consider the effect of noise reduction on the linear and nonlinear measure of dependencies. We also use singular spectrum analysis as a powerful method for filtering financial series. We compare the results with those obtained by ARMA and GARCH models as linear and nonlinear methods for filtering the series. We also examine the findings on an artificial data set namely the Hénon map.
\end{abstract}

(c) 2009 Elsevier Ltd. All rights reserved.

\section{Introduction}

During the last few years the analysis of financial time series has received increasing attention. Many researchers have discovered evidence for the possibility that the financial markets may be nonlinear dynamical systems, with important implications in the Efficient Market Hypothesis. Several researchers, by using different statistical tests, have mentioned evidence of non-independently and identically distributed (IID) behavior and nonlinear dependence in financial time series [1-9].

Several measures have been used to calculate the degree of independency or dependency. The most known measure to calculate dependency between two random variables is the coefficient of linear correlation, but its application requires a pure linear relationship, or at least a linear transformed relationship. However, this statistics may not be helpful in determining serial dependence if there is some kind of nonlinearity in the data [10,11].

Urbach [12] defends a strong relationship between entropy, dependence and predictability. This relation has been studied by several authors [11-14]. It has been shown that a measure based on the mutual information, which captures linear and nonlinear dependencies, without requiring the specification of any kind of model of dependence, is better than the linear correlation coefficient to measure serial correlation of several stock market indices [10-14].

Recently, two new methods have been developed to measure long-range correlations in nonstationary fluctuating series; the detrended fluctuation analysis $[15,16]$ and the detrended moving average method $[17,18]$. These methods detect

\footnotetext{
* Corresponding author at: Institute for Studies and Research (ITST), Tehran, Iran.

E-mail address: hassanih@cf.ac.uk (H. Hassani).
} 